\title{
Lipid-Protein Microinclusions in the Morphological Structures of Organelle Membranes Studied by Fluorescent Confocal Microscopy
}

\author{
Michael Yu. Chernyshov ${ }^{*}$, Vadim N. Nurminsky², Natalia V. Ozolina² \\ ${ }^{1}$ Presidium of Irkutsk Scientific Center, Siberian Branch, Russian Academy of Sciences, Irkutsk, Russia \\ ${ }^{2}$ Siberian Institute of Plant Physiology and Biochemistry, Siberian Branch, Russian Academy of Sciences, Irkutsk, Russia \\ Email: ^Michael_Yu_Chernyshov@mail.ru
}

How to cite this paper: Chernyshov, M.Yu., Nurminsky, V.N. and Ozolina, N.V. (2017) Lipid-Protein Microinclusions in the Morphological Structures of Organelle Membranes and Studied by Fluorescent Confocal Microscopy. Advances in Biological Chemistry, 7, 42-59.

https://doi.org/10.4236/abc.2017.71003

Received: December 8, 2016

Accepted: February 20, 2017

Published: February 23, 2017

Copyright $\odot 2017$ by authors and Scientific Research Publishing Inc. This work is licensed under the Creative Commons Attribution-NonCommercial International License (CC BY-NC 4.0).

http://creativecommons.org/licenses/by-nc/4.0/

\begin{abstract}
Peculiar properties of morphological structures of organelle membranes were studied by fluorescent confocal microscopy. The list of objects in our experiments was represented by mitochondria, chloroplasts and vacuoles. During this study, identification of lipid microinclusions having the form of such lipid-protein structural microformations as lipid-protein microdomains, vesicles and membrane tubular structures (cytoplasmic transvacuolar strands and nanotubes) located in organelle membranes or bound up with them was conducted. Such membrane probes as laurdan, DPH, ANS and bis-ANS were used. Comparison of fluorescence intensity of these membrane probes was conducted. This investigation of the morphological properties of lipid-protein structural microformations was accompanied with analysis of 1) the phase state and 2) dynamics of microviscosity variations in the membrane elements of isolated plant cell organelles. Distributions of laurdan fluorescence generalized polarization (GP) values for the membrane on the whole and for the intensively fluorescing membrane segments were obtained. It was discovered that the microviscosity of intensively fluorescing membrane segments essentially differed from the microviscosity of the rest part of the membrane. In conclusion, some results of the study of peculiar properties of lipid-protein structural microformations related to the structure of organelle membranes and the discoveries made in this investigation are discussed.
\end{abstract}

\section{Keywords}

Identification of Lipids, Morphological Structure of Cell Organelles, Morphological Structure of Vacuolar Membrane, Fluorescent Confocal Microscopy, Fluorescent Probes, Lipid-Protein Structural Membrane 
Microinclusions, Types of Microformations Identified, Lipid-Protein

Microdomains, Lipid-Protein Membrane Nanotubes, Vesicles,

Cytoplasmic Strands, Microviscosity

\section{Introduction and Problem Statement}

As obvious from earlier publications of several authors, the cell membrane is characterized by mobile lateral nonhomogenieties of its lipid content represented in the form of microinclusions [1] [2] [3]. These microinclusions may be studied in experiments presuming 1) visualization of the cell membranes with finding out examples of such microinclusions and 2) investigation of their characteristics.

The stimulus for our investigation was absence of plausible knowledge about the structure and the functions of lipid-protein microformations in cell organelles, as well as the interest to the unusual membrane microinclusions registered in our observations earlier [3]. In particular, the domain structure of vacuolar membranes has not been plausibly scanned and investigated before. Meanwhile, some authors are in the position of attributing them rather specific functions (say, trafficking, signaling, etc.), while doing it prior to plausible registrations and detailed investigations of such microformations. The authors are sure that any intuitive evidence in science is insufficient. The point is that the study of microformations has to be continued. The reliable results are needed by biology and medicine.

Understanding of the structure and the true functions (role) of such microformations in membrane activity is essential not only from the viewpoint of advancements in biology in principle but also in the aspect of problems of cell medicine and pharmacology. For example, it is known that there are drugs for heart attacks and elevated blood pressure, which are oriented to either stimulation or blockage of ion transmembrane activity in organelles. As far as investigations of mitochondria are concerned, it is known that efficient are translational techniques of mitochondrial disfuction diagnostics in patients suffering chronic myocardial ischemia or brain ischemia. The same concerns vacuoles, in which the potassium transport is driven by vacuolar $\mathrm{H}^{+}$-ATPase (V- $\mathrm{H}^{+}$-ATPase).

On the first stage of our investigation, we planned to identify and study lipidprotein microinclusions in organelle membranes considered as the membrane nonhomogenieties having the form of diverse microformations in which lipids were seen to be quantitatively dominant.

The list of objects of our experiments was represented by mitochondria, chloroplasts and vacuoles. It is expedient to note that vacuoles are the objects whose investigation was ignored for a long time in connection with the complexity of work with such objects, furthermore, considered as inessential. Understanding of the role of this organelle changed after some recent publications [3] [4] [5].

The set of lipid-protein structural microinclusions expected to be revealed and registered in the experiments included lipid-protein microdomains (LPMs), ve- 
sicles, cytoplasmic strands and lipid-protein membrane nanotubes. The nature these lipid-protein nonhomogenieties was to be understood.

One of the starting points was the assumption that lipid-protein microformations are formed around definite proteins and enriched with sterols, glycosphingolipids and lipids with saturated fatty acids [3] [6] [7].

The first object of our experiments was represented by vacuoles. Our interest to vacuoles was conditioned by the fact that $\mathrm{V}-\mathrm{H}^{+}$-ATPase determines homeostatic processes in kidney cells, cardiac muscle cells, etc. For example, in highly metastatic breast cancer cells, the potassium transport is driven by V- $\mathrm{H}^{+}$-ATPase [8]. It was registered that all cancer cells had substantially higher $\mathrm{V}-\mathrm{H}^{+}$-ATPase activity than MCF-10A cancer cells or other breast cancer cell lines. This transport protein capable of maintaining intracellular ion homeostasis is a more obvious target for anticancer therapy than $\mathrm{Na}^{+}-\mathrm{K}^{+}$. Furthermore, V- $\mathrm{H}^{+}$-ATPase activity of plant vacuoles is probably similar to that in animal organelles, and it has been investigated earlier, for example, in the aspect of stress [9] [10] [11] [12].

Peculiar properties of membrane morphological structures of plant cell organelles were studied with the aid of fluorescent confocal microscopy. The cell of Beta vulgaris $\mathrm{L}$. chosen is characterized by the presence of a large vacuole, which can occupy a substantial part of its volume. The principal vacuolar functions presume participation in cytosol ion homeostasis, in processes of storage of primary/secondary methabolites, osmotic regulation, detoxification of xenobiotic toxins, formation of cell immune response reactions (for example, methabolitic reactions to toxins) and also in processes of apoptosis [13] [14].

A plant cell vacuole represents an actively functioning organelle capable of dynamic morphological transformations. The plant cell vacuolar system possesses a complex architectonics. It is known, for example, that vacuolar membranes are mediators of transmembrane transport. Some specialists register vacuolar membrane invaginations accompanied with separation of vesicles [15] [16] [17] [18]. It has been discovered that there are numerous cytoplasmic strands starting in the vacuolar membrane and going beyond its limits [17] [19]. The relations of vacuole with cytoskeleton elements [18] (for example, with actin microfilaments) can be traced [15] [20]. Furthermore, when the cell prepares for mitosis, transformations of the vacuolar membrane structure may take place [15]. Any vacuole is known to be more active, when the cell consumes various compounds in the process of pinocytosis [4]. Any cell actively reacts to osmotic stress conditions [16].

It has been shown already in the 1990s, the vacuolar membrane is characterized by definite order of the lipids, which exist in it in the form of some formations in the liquid lipid bilayer and some domains bound up with membrane proteins. Polar lipids containing unsaturated fatty acid residues (chains) dominate in the vacuolar membrane. This domination ensures membrane's high elasticity and low microviscosity. The fact of existence of lipid-protein microdomains in plant vacuolar membranes was confirmed in [3] [5]. These are characterized by relatively dense packaging of lipids with respect to the rest part of the 
vacuolar membrane. Still the ultrastructural peculiar properties of the vacuolar membrane as well as its role in adaptation to stress effects remain underinvestigated [21]. In order to clarify the hypotheses, analysis with the application of the technique of fluorescent confocal microscopy has been conducted.

The objectives of this investigation presumed experiments in order to:

1) visualize the organelle membranes and hence identify such lipid-protein structural formations as lipid-protein microdomains (LPMs), vesicles, cytoplasmic strands and nanotubes located in plant cell organelle membranes or bound up with them;

2) assess a) the phase states and b) the dynamics of local microviscosity and its variations in the organelle membrane elements of isolated plant cell organelles.

Some results of identification of some details of the vacuolar membrane morphology as well as its structural peculiarities in the norm and under osmotic stress are discussed.

\section{Materials and Methods}

Our experiments were conducted on isolated vacuoles (tonoplast) of Beta vulgaris L. dormant storage beet roots, variety Bordeaux (the seeds being obtained from Sibirskii Sad, Inc., Novosibirsk, Russia, and planted on the experimental field of Siberian Institute of Plant Physiology and Biochemistry, Siberian Branch of RAS) stored at +4 to $5^{\circ} \mathrm{C}$. Tonoplast from the beet root tissues was isolated with the aid of a modified microobject technique described in [22] in an isolation solution containing $300 \mathrm{mM} \mathrm{KCl}, 10 \mathrm{mM}$ EDTA, $25 \mathrm{mM} \mathrm{NaH}{ }_{2} \mathrm{PO}_{4} / \mathrm{KOH}$, $\mathrm{pH}$ 8.0, $\beta$-alanine ( $650 \mathrm{mOsm} \cdot \mathrm{kg}^{-1} \mathrm{H}_{2} \mathrm{O}$ ) (see e.g. [3]). The result of isolation was purified.

In order to visualize isolated organelle (vacuolar) membranes and their lipidprotein structural nano-formations, to assess the membrane phase states and the bilayer microviscosity we applied confocal microscopy (confocal fluorescence scanning laser microscope MicroTime 200 (PicoQuant GmbH, Germany) and several fluorescent probes.

Scanning of isolated organelles with the use of fluorescent confocal microscopy in our experiments was intently conducted at the normal room temperature, what was of interest for us. The objects were registered at various distances from the glass basement. Each time, in the upper part of an isolated vacuole lying on the basement, we observed a large membrane segment and registered the effect of highly intensive fluorescence of some of the membrane segments.

Molecules of the probes used are capable of inserting between the molecules of the objective substance. The following fluorescent probes were used: filipin (having affinity to sterols); laurdan (or 2-(dimethylamino)-6-dodecanoilnaphtaline), a lipophilic probe actively fluorizing in contact with hydrophobic domains; ANS (8-anylino-1-naphtalensulphonic acid) known to have affinity mainly to proteins; bis-ANS and diphenilhexatrien (DPH) (Sigma-Aldrich, USA). Unfortunately, we had no probes having affinity to sphingolipids. Other chemically pure reagents were $\mathrm{KCl}$, EDTA, $\mathrm{NaH}_{2} \mathrm{PO}_{4}, \beta$-alanine, etc. (all manufactured by Che- 
mreactivesnab, Ltd., Russia).

One of the simplest techniques for assessment of membrane's lipid component microviscosity presumed measurement of fluorescence generalized polarization (GP) characteristic of a given fluorescent probe, when this probe was introduced into the membrane's lipid component. In assessment of microviscosity we used a technique based on computing laurdan fluorescence GP values at each pixel of the image light part. Such a technique presumed constructing a GP value distribution histogram. The histogram represented arithmetic mean values and their SD values. The statistical data processing was conducted with the aid of Excel.

Laurdan is fluorescing mainly in two spectral domains depending on the system's fluidity (i.e. depending on the presence of free water molecules in the membrane's lipid component) [3] [23]. Its extensive application is conditioned by the assumption that locations of its emission maxima are determined by the relationship existing between liquid-ordered and liquid-disordered membrane domains. This relationship may be assessed by the value of the probe's GP. To the end of binding laurdan molecules and the vacuolar membrane molecules, this probe (diluted in methanol down to the final concentration of $10 \mu \mathrm{M}$ ) is added to the suspension of isolated vacuoles. The suspension is incubated at $20^{\circ} \mathrm{C} \pm 2{ }^{\circ} \mathrm{C}$ during $10 \mathrm{~min}$ and observed via the confocal microscope. The GP value for each pixel of the image obtained is computed automatically with the aid of special software [24]. Next, all the GP values are added, and the mean value and the SD are computed. Furthermore, the software constructs a GP value distribution histogram for the pixels characterized by some fluorescence intensity exceeding a definite threshold. In the process of computing GP values, the importance of such a threshold is conditioned by the need of taking account of only brightly fluorescing pixels corresponding to the membrane and not taking account of the background. The technique of computing GP values for the membrane probe fluorescence corresponding to the accepted confocal microscopy technology can be found in [3].

In our experiments with the use of ANS (or bis-ANS) and DPH, the probe was added to the suspension of isolated vacuoles in concentration of $10 \mu \mathrm{M}$ in $10 \%$ dimethyl sulphoxide and tetrahydrophuran, respectively. After $30 \mathrm{~min}$ of incubation the suspension was viewed by the confocal microscope, the probe's fluorescence intensity being quantitatively assessed. The value of fluorescence intensity was determined on account of the number of photons registered from a given segment of a sample under scrutiny. Fluorescence lifetime imaging microscopy (FLIM) technique was used. A single-photon avalanche diode (SPAD) built into the confocal microscope was used to register single photons.

\section{Results}

Visualization of the vacuolar membrane structures was conducted with the use of fluorescent probes (filipin, laurdan, ANS, bis-ANS, DPH).

Figure 1 shows the images of isolated vacuoles stained with filipin $(5 \mu \mathrm{M})$, Figure 1(a), Figure 1(b) corresponding to optical sections in the central part of 
the vacuole at a distance of $5 \mu \mathrm{m}$ from the basement; Figure 1(c)-in the upper part of the vacuole. Here solid arrows indicate to large sterol-containing membrane segments; dotted arrows-to sterol-deficient membrane segments. Figure 2 shows the images of isolated vacuoles stained with filipin in different concentrations $(1 \mu \mathrm{M}$ Figure 1(a); $5 \mu \mathrm{M}$ Figure $1(\mathrm{~b})$ and $15 \mu \mathrm{M}$ Figure 1(c)), optical sections being made in the upper parts of the vacuole.

In the process of visualization and investigation of our preparations with isolated vacuoles, we-in all the cases-distinctly registered lipid-protein membrane elements: LPMs revealed by filipin (Figure 1(c) and Figure 2(b) and Figure 2(c)), vesicles revealed by laurdan (Figure 3(b)), and also so called membrane tubular structures (MTSs): cytoplasmic transvacuolar strands (earlier discussed in [25] [26]), which had the form of threads (Figure 3(a)), and nanotubes (Figure 4) located in plant cell organelle membranes or bound up with them.

Comparison of fluorescence intensity for such membrane probes as laurdan, DPH, ANS and bis-ANS was conducted (see Figure 5). We discovered that vacuolar membranes isolated in the solution (isotonic with respect to that of the cell sap of roots) were stained nonhomogeneously. This was especially obvious from the results of scanning the upper (with respect to the basement) surface of
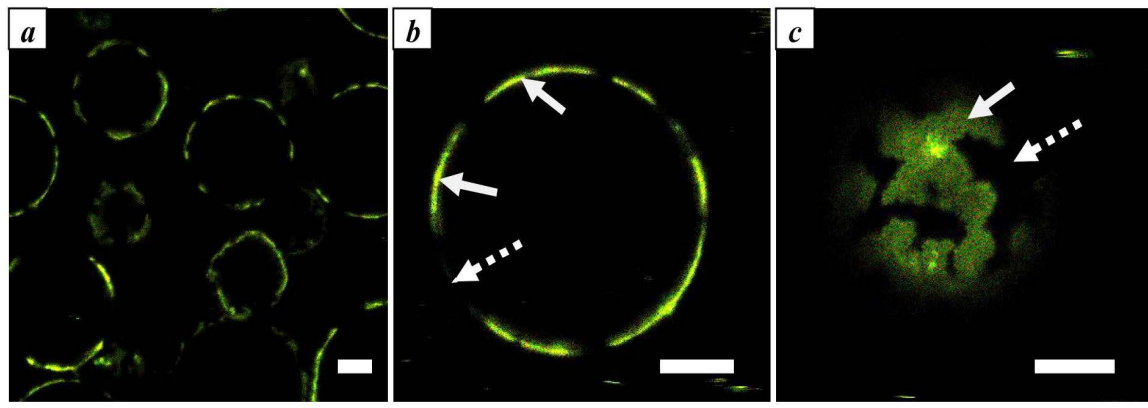

Figure 1. Images of isolated vacuoles stained with filipin (5 $\mu \mathrm{M})$, (a), (b) corresponding to optical sections made in the central part of the vacuole at a distance of $5 \mu \mathrm{m}$ from the basement; (c) in the upper part of the vacuole. A solid arrows-large sterol-containing membrane segments; dotted arrows-sterol-deficient membrane segments; Ob. 60×; scale bars $-0 \mu \mathrm{m}$.
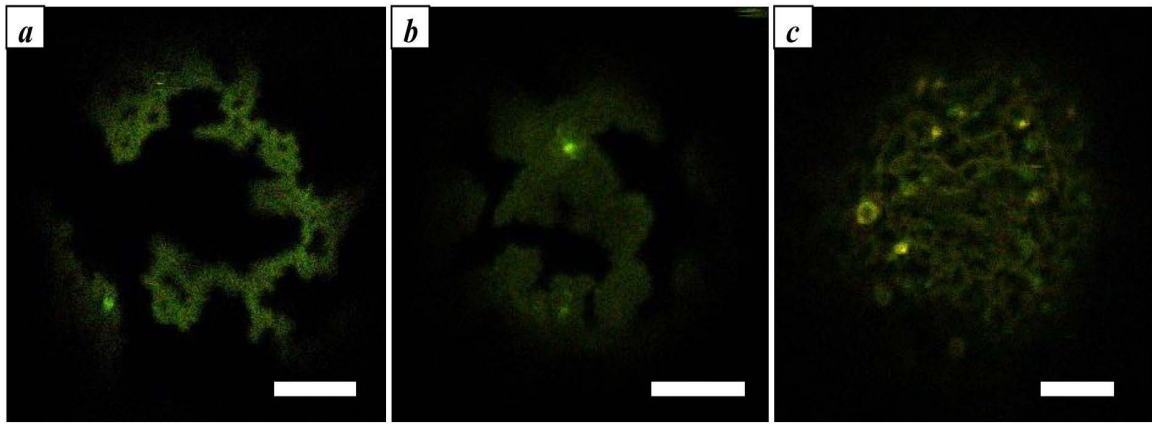

Figure 2. Images of isolated vacuoles stained with filipin in different concentrations (1 $\mu \mathrm{M}(\mathrm{a}) ; 5 \mu \mathrm{M}$ (b) and $15 \mu \mathrm{M}$ (c)); optical sections are made in the upper parts of the vacuole; Ob. $60 \times$; scale bars $-10 \mu \mathrm{m}$. 

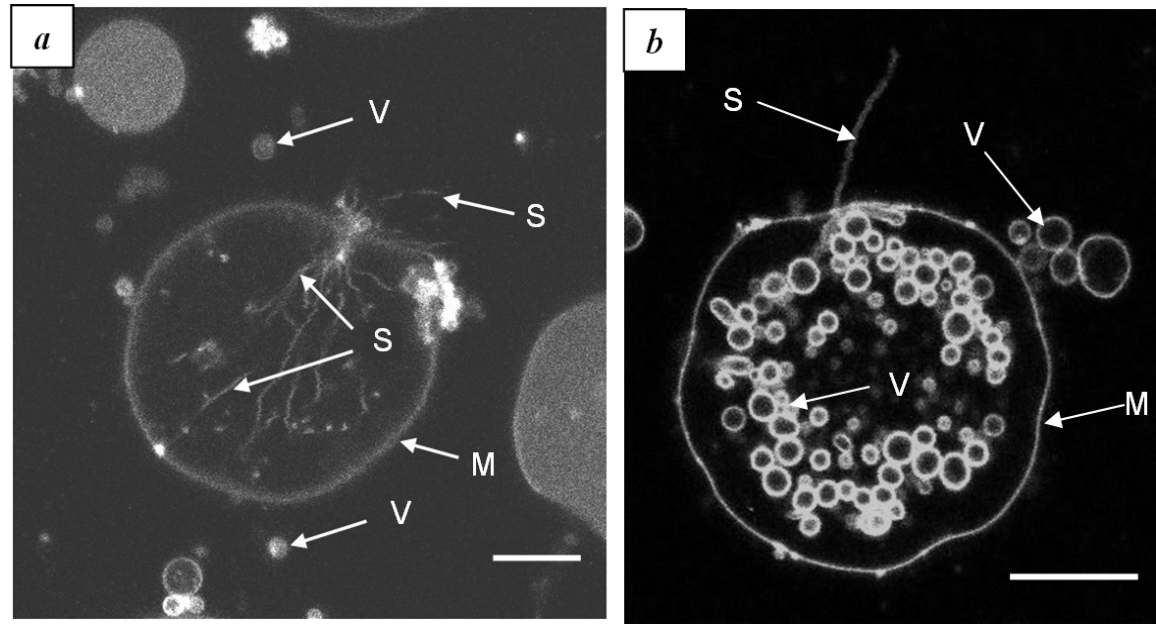

Figure 3. Images of isolated vacuoles stained with fluorescent probes ANS (a) and laurdan (b). Optical sections are made at a distance of $5 \mu \mathrm{m}$ from the basement. $V$-vesicles, $M$-membrane, $S$-transvacuolar strands. Ob. $60 \times$, scale bars-10 $\mu \mathrm{m}$.

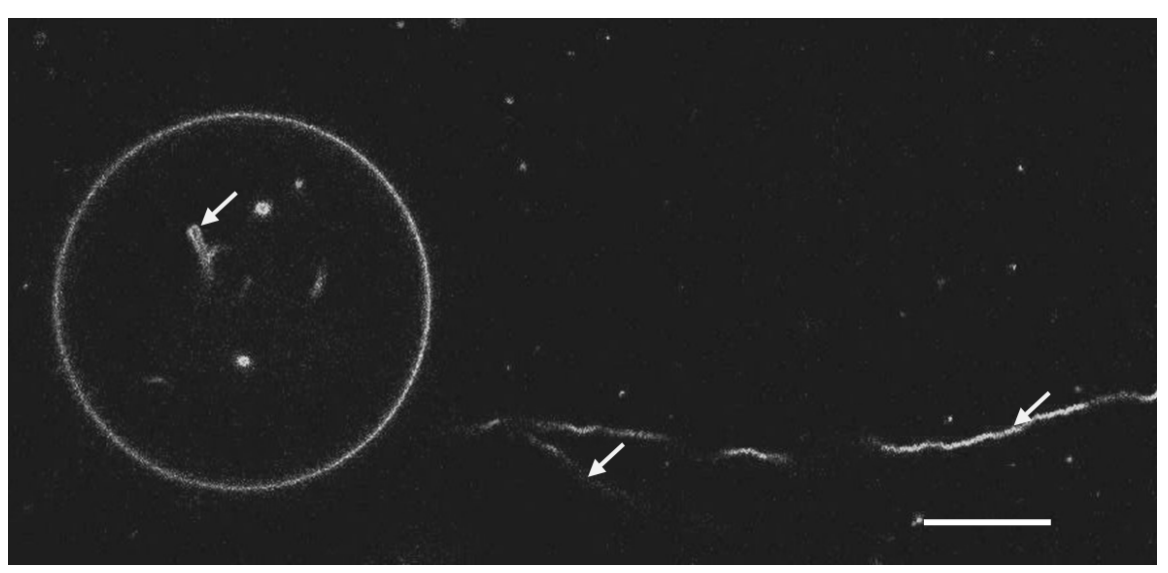

Figure 4. Membrane nanotubes (pointed by arrows) inside and outside the vacuole. Probe-laurdan. Ob. $60 \times$, scale bar-10 $\mu \mathrm{m}$.

the vacuole, when a substantial membrane surface occured in the microscope's focal plane. Brightly fluorescing segments and tracks inside the vacuolar membrane were registered in the confocal images when all the above probes were used. These were characterized by an increased fluorescence intensity of the probes (assessed to be 10 times as large as the fluorescence intensity of the close parts of the membrane). Fluorescence intensity of the probes was not the same not only in different parts of the membrane but also for various vacuoles (according to our data, fluorescence intensity of different vacuoles differed by 2 to 3 times). Noteworthy, we did not register any brightly fluorescing segments in the preparation sites, where there were no vacuoles.

On account of the information that ANS (Figure 5(c)) and bis-ANS (Figure 5(d)) have a more expressed affinity to proteins, it is possible to assume that these tracks represent membrane segments characterized by an elevated content of proteins, which get to the zone of microscopic scanning. 


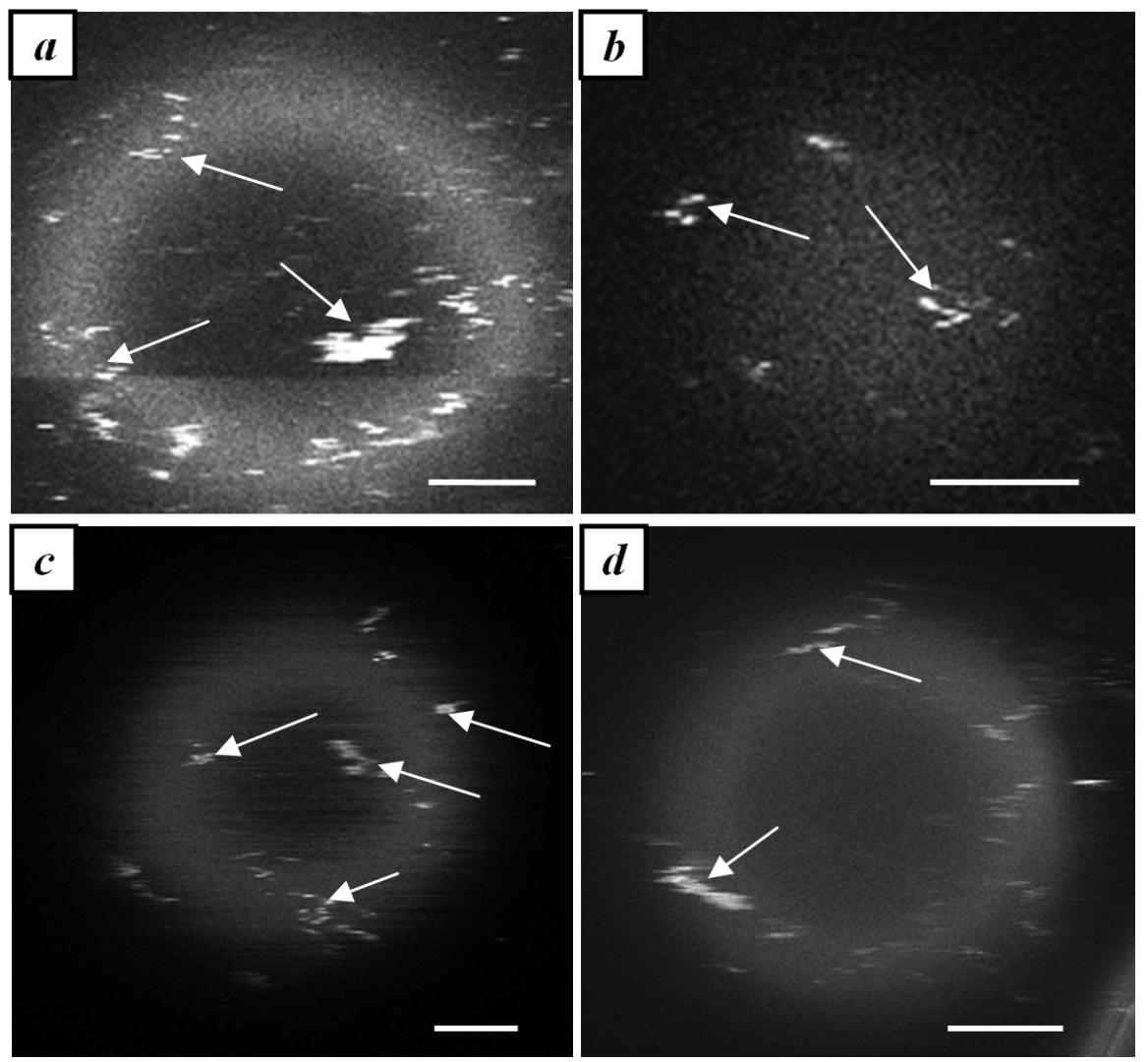

Figure 5. Zones of intensive fluorescence (pointed by arrows) in the top part of an isolated vacuole: (a) laurdan; (b) DPH; (c) ANS; (d) bis-ANS. Fluorescence intensity sites of the segments and tracks pointed by arrows exceed fluorescence intensity of the rest membrane parts approximately by 10 times. Ob. $60 \times$, scale bar $-5 \mu \mathrm{m}$.

\section{Discussion}

It is known that a plant cell vacuole represents an active organelle, which may acquire some complex architecture in the process of preparing for cell division.

In recent years, the attention to investigations related to biological membranes has been attracted to the structures of cylindrical form, i.e. to so called "membrane nanotubes", having the diameter from several decades to hundreds of nanometers [2]. This attention is explained by the fact that many cellular processes (for example, such processes as endocytosis and fusion of cells) are bound up with formation of unusual membranes and/or trans-membrane structures. Membrane tubular structures (MTSs) looking similar to the ones registered in our experiments were earlier identified in cell extracts [25]. Only one simple attempt to explain the formation of MTSs in the process of endocytosis is known [27] [28]. It is desirable to obtain a plausible explanation of the mechanisms of formation of membrane nanotubes in cells and in their organelles.

Functions of MTSs have to be understood. As a result of investigations conducted on some natural and artificial membrane structures it has been demonstrated that MTSs form a ramified transport tubulovesicular network, contractile (motor) cytoskeleton proteins participating in its formation [25] [28]. So, MTSs are responsible for the formation of tubulovesicular networks. Furthermore, 
there are some unconvincing hypotheses of their participation in the processes of intracellular and intercellular transport [29] [30].

As discovered in our investigation, the lengths of MTSs vary rather widely. These can reach more than $300 \mu \mathrm{m}$, what is 5 times as large as the mean diameter of a vacuole, $60 \mu \mathrm{m}$ (Figure 3). The technique of zeitraffer scanning has allowed us to find out that the vesicles bound up with MTSs may move in the space with respect to the isolated vacuoles fixed on the basement. Such a displacement of vesicles bound up with the membrane tube in the process of their transition may be explained by either motions of vesicles along an MTS or displacement of such an MTS (with the vesicles fixed on it) on the whole. In the latter case, variation of the MTS's length possibly takes place.

It has been demonstrated in the experiments conducted on giant unilamellar vesicles (GUV) that formation of MTSs depends on both the temperature and the content of the lipids [1]. On the basis of model experiments conducted on artificial membrane structures, Y. Sakuma et al. (2013) assumed that formation of such structures may be bound up with the balance in the tension inside the vesicle membrane (spontaneous tension) and, possibly, also with asymmetric distribution of inverse-cone-shaped lipids (lipids having the molecule forming a sort of inverse-cone in the internal and external layers of the vesicle membrane).

For example, it has been demonstrated in experiments with GUV that-in course of formation of MTSs-there takes place a process of dynamic clusterization of such a motor protein as kinesin [31]. There are also discussions in the literature related to the probability of influence of cytoskeleton elements upon the membrane's structure. These data were obtained on both artificial and natural membranes. For example, it was shown in the studies of GUVs that the actin networks bound up with the membrane may themselves determine the time and the place of membrane microdomain formation [32]. It has also been demonstrated that trans-membrane proteins in animal cells (together with cytoskeleton proteins) are capable of integrating of membrane lipids into large microdomains under definite conditions [33].

It is logical to assume, firstly, that unique membrane structures (vesicles (Figure 3(b)) and nanotubes (Figure 4)) observed in our experiments may participate in implementation of some definite cellular functions, say, the transport function. Many authors tend to discuss such functions as trafficking, signaling, etc., but unfortunately, on the basis of model experiments with artificial membranes. So, their assumptions has to be proved for natural membranes, in vivo.

Secondly, it is natural to state that MTSs observed in our experiments have a lipid-protein nature. Indeed, these are well-stained by lipid affinity membrane probes and possess the microviscosity close to that of the vacuolar membrane. Microviscosity was assessed via GP values of laurdan fluorescence, the data can be found in our publication [3]. Thirdly, on account the data on existence of the dynamical relationship between 1) the cytoskeleton and the vacuole [15] [20] and 2) the cytoskeleton and the MTSs [25] [28], we have assumed that the structures observed in our experiments are related to the vacuolar cytoskeleton elements. 
Tubular-like transvacuolar strands inside vacuoles were earlier registered in membranes of some cells [16] [34]. Furthermore, transvacuolar strands in cell vacuoles in the culture of Arabidopsis thaliana protoplasts were discovered to possess a rather complex structure. Their transformations are supposed to be bound up with actin filaments [15].

Vesicular structures gemmating from the vacuolar membrane and the tubular-like transvacuolar strands inside vacuoles observed were earlier discovered in membranes of other plant cells [16] [34]. Effects of influence of osmotic stress upon the membrane's state and upon formation of vesicles are, no doubt, of interest. Formation of vesicles has been described for the onion epidermis cells under osmotic stress [16]. It is characteristic that in this case the exocytosis vesicles do not completely separate from the plasma membrane, but remain linked to it by a thin thread going from the membrane. Such a connection between the vesicle and the membrane is supposed to be important from the viewpoint of successful process of subsequent deplasmolis [16].

It has earlier been discovered that in some cases, in the process of endocytosis, the vesicles do not completely separate from the cytoplasmic membrane, but remain connected with it via a thin $(\sim 20 \mathrm{~nm})$ membrane nanotube [35]. Such decomposition is natural, say, in cases of osmotic influences. In our case, such a decomposition took place under either hyper- or hypoosmotic conditions. An explicit example of the result of vacuolar membrane decomposition into many vesicles under hyperosmotic stress is shown in Figure 3(b), where one can see an image obtained at the moment, when the upper part of the vacuole has already burst (and is completely absent), and the focal plane of the confocal microscope is $5 \mu \mathrm{m}$ from the basement, where the ring of vesicles lying on the bottom is observed. As obvious from the observations of the same preparations in dynamics, the breach of the membrane's integrity is not always entailed with decomposition of the vacuole. In cases of occurrence of local membrane defects, the vacuole looses some part of its internal content, so, some of the membrane nanotunes could proceed out of the vacuole. After that, the place of the defect may be "cured", and the integrity of the membrane restored.

In our observations we discovered the cases of complete decomposition of membrane vacuoles in dynamics. This decomposition was accompanied with mass formation of vesicles instead of the membrane (Figure 6). Gemmation of vesicles from the vacuolar membrane observed took place sooner in the form of either endocytosis or exocytosis, and in these cases, the vesicles were observed both inside and outside the decomposed vacuole.

Analysis of the data related to fluorescence of membrane probes allows one to draw preliminary conclusions on the phase state (and density) of the membrane's substance [36].

In our case, mean GP values reflecting 1) fluorescence of the domains characterized by an increased fluorescence intensity and 2) fluorescence of the membrane on the whole were plausibly different $(-0.22$ and -0.04 , respectively, $p<$ 0.05 , the Mann-Whitney criterion). Meanwhile, the character of distribution of 


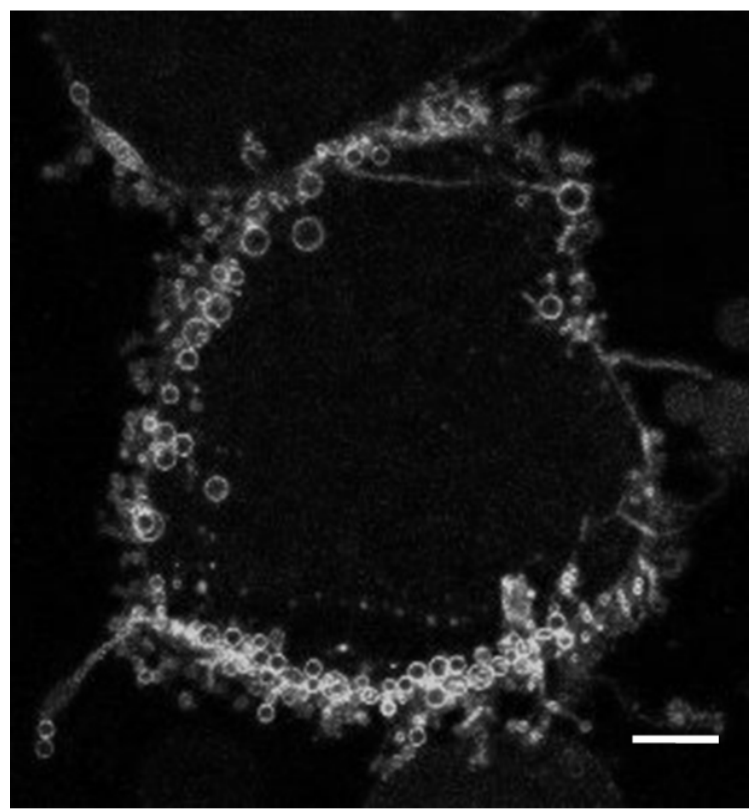

Figure 6. A result of decomposition of an isolated vacuolar membrane into a set of vesicles under hyperosmotic stress. Probe-laurdan; optical are sections at a distance of $5 \mu \mathrm{m}$ from the basement. Ob. $60 \times$, scale bar $-10 \mu \mathrm{m}$.

GP values in case of bright segments was different with respect to the distribution of GP values for the total membrane (Figure 7). It was possible to reveal at least 3 separate peaks on the histogram showing the distribution of laurdan fluorescence GP values in the domains characterized by higher fluorescence intensity (Figure 7, grey columns). There were spectral domains of GP values among three above peaks, which corresponded to 1) the mean density characteristic of the liquid disordered (more liquid) state and 2) a denser substance state (probably, liquid ordered state), the maxima of GP values being $-0.5,-0.2$ and 0.35 , respectively). As obvious from the histogram in Figure 7, laurdan fluorescence GP values for the vacuolar membrane in the norm have a distribution close to the normal one and vary within the band from -0.6 to 0.4 (the maximum being at -0.1), what corresponds to the liquid-crystalline state of membrane lipids (see also [23]).

Analysis of the data on fluorescence of membrane probes allows one to assess also the microviscosity of the lipid membrane's matrix [3] [23] [36]. For example, membrane's microviscosity may be assessed by variations in the fluorescence spectra [37]. In our experiments, we have obtained distributions of laurdan fluorescence GP values for the vacuolar membrane on the whole and for intensively fluorescing membrane segments. In such segments, microviscosity may substantially differ (be either increased or decreased) from the rest part of the membrane. It has been ascertained that the vacuolar membrane is stained non-homogeneously. Furthermore, the domains are characterized by an explicit affinity to definite membrane probes [3]. Such segments may probably represent the clusters characterized by intensive processes of lipid-protein formation, in 
which proteins play the dominant role [5] [38]. It also known that such segments are characterized by high mobility [39] [40]. These facts have been confirmed in our investigation. The tracks registered in Figure 5(c) and Figure 5(d) represent membrane segments characterized by an elevated content of proteins. Such segments are supposed to give evidence of high mobility of the respected mictoinclusions. In cases of repeated scanning (time-lapse), these were registered in shifted positions (see Figure 8). It is logical to suppose that the vacuolar membrane segments showing higher intensity of fluorescent marking with membrane probes are characterized by higher density of proteins in the membrane.

It may be supposed that normal functioning of the membrane (i.e. normal execution of its physiological functions) is possible only in case of sufficient mobility of its structural components. Such a biophysical parameter as membrane's microviscosity is mainly limited by its lipid components. In turn, microviscosity

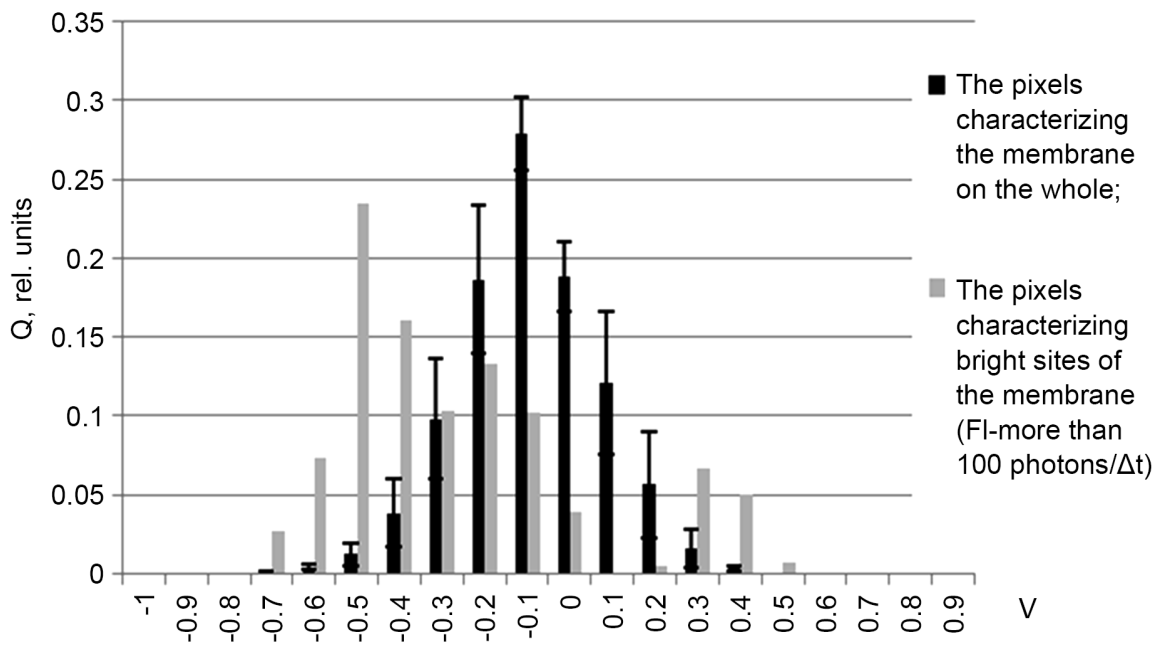

Figure 7. The distribution of GP values for fluorescence of laurdan placed in the lipid component of vacuolar membrane. The abscissa axis: V-GP values; the ordinate axis: Qnumber of pixels on the videoimage, which are characterized by definite fluorescence intensities (FI). Relative units are understood as the ratio of the number of pixels, for which the GP values computed are within some definite interval, to the total number of videoimage pixels characterized by FI, which is in excess of a definite limit (the GP analysis technique can be found in "Materials and methods")
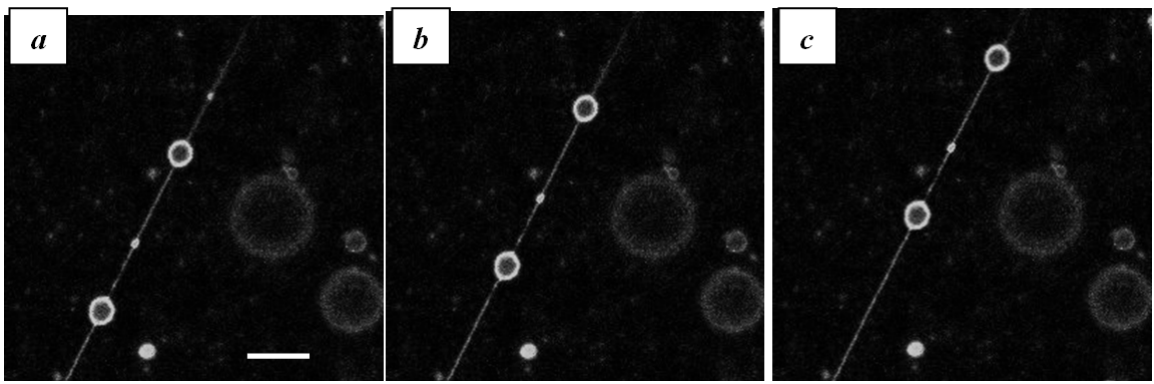

Figure 8. Displacement of vesicles bound up with the membrane tube in the process of their transition. (a)-(c) images obtained in the process of scanning one of the segments intermittently at equal time intervals ((a) $0 \mathrm{sec}$, (b) $30 \mathrm{sec}$, (c) $60 \mathrm{sec}$ ). Probe laurdan. Ob. $60 \times$, scale bar $-5 \mu \mathrm{m}$. 
determines the rate of translational and rotational motions of the molecules (including membrane's protein molecules) [41]. Possibly, that is why variations of microviscosity are considered as an important physiological reaction of the cell and its membrane, which is conditioned by processes of adaptation of the organism to external factors [42]. Variations of membrane's microviscosity are also assumed to incur variations in membrane's permeability as well as variations in the activity of the membrane proteins (existing in the forms of enzymes, ion channels, receptors) [43]. This, in turn, leads to the development and (or) intensification of regulatory reactions oriented to organism's adaptation to unfavorable environmental conditions.

\section{Conclusions}

In course of the first stage of the present investigation 1) visualized were the vacuolar membranes, 2) mobile lateral nonhomogenieties of its lipid content were identified in the form of such lipid-protein structural formations as lipid-protein miscodomauns, vesicles, cytoplasmic strands and nanotubes located in the plant cell, in vacuolar membrane or bound up with the latter; 3) distribution of lipids on the surfaces and inside above structural formations was assessed; 4) assessed were the phase states and the dynamics of local miscoviscosity and its variations in the zones of vacuolar membrane elements.

On account of the facts revealed in our investigation it is possible to state the following.

1) Application of filipin as a sterol binding probe has given us the posiibility to reveal that an isolated vacuolar membrane is stained nonhomogeneously (Figure 1). This is obvious in the processes of scanning the central part of vacuoles (Figure 1(a), Figure 1(b)) and its upper (with respect to the surface layer) surface (Figure $1(\mathrm{c})$ ). In the latter case, a substantial membrane surface remains in the focal plane of the microscope. This allows to assume that there are two types of large domains on the membrane: sterol-containing and steroldeficient (containing sterols in very small quantities) ones. Furthermore, small sterol-enriched zones were registered.

2) A dependence of the character of interaction between filipin and membrane sterols on the filipin concentration was revealed (Figure 2). In is obvious that the domain organization of location of sterols in tonoplast is retained within the band of filpin concentrations of 1 to $5 \mu \mathrm{M}$ (Figure 2(a), Figure 2(b)). Meanwhile, this domain organization is violated (and acquires a complex networktype character) in case of increase in the concentration of filipin (Figure 1(c)). This is probably bound up with some nonspecific interaction of filipin molecules with the membrane sterols under high filipin concentrations. Deeper undestanding of these interactions between membrane sterols and filipin necessitates some additional investigations.

The present investigation has given the following discoveries.

- Vacuolar membranes isolated in the solution (isotonic with respect to that of the cell sap of roots) are stained nonhomogeneously. This is especially ob- 
vious from the results of scanning the upper (with respect to the basement) surface of the vacuole, a substantial membrane surface occurs in the microscope's focal plane. In this case, fluorescence intensity of the probes is not the same not only in different parts of the membrane but also for various vacuoles. According to our data, fluorescence intensity of different vacuoles differs by 2 to 3 times.

- Membrane clusters rich in lipids are characterized by an elevated density of packaging of protein and lipid molecules, and by microviscosity, which is higher than that of the rest (surrounding and more liquid) part of the membrane. Membrane nanotubes were observed both inside and outside the vacuoles, and in case of scanning intermittently at equal time intervals observed were transitions of vesicles with the nanotube (Figure 5).

- An effect of vacuolar membrane decomposition into many vesicles under hyperosmotic stress (shown in Figure 3(b)).

- An effect of complete decomposition of membrane vacuoles in dynamics, which is accompanied with mass formation of vesicles in the place of membrane, has been demonstrated (Figure 4). Gemmation of vesicles from the vacuolar membrane observed in many experiments took place in the form of either endocytosis or exocytosis. In these cases, the vesicles were observed both inside and outside the decomposed vacuole.

Therefore, our investigation of the lipid content and distribution, morphological properties, phase states and also microviscosity of membrane elements of isolated plant cell vacuoles with the aid of confocal microscopy has allowed us to confirm the fact of formation of such structural lipid-protein nonhomogenieties in the system of vacuolar membranes as lipid-protein microdomains, and such membrane tubular structures (MTSs) as vesicles, cytoplasmic strands and lipid-protein membrane nanotubes.

These nonhomogenieties were revealed both inside and outside the vacuole. Furthermore, in the process of scanning after equal time intervals it was possible to observe transmissions of vesicles side by side with membrane tubular structures. In the process of scanning the upper part of the isolated vacuole we observed separate segments characterized by high fluorescence intensity. Such segments may represent clusters of lipid-protein formations with prevalence of proteins in their content. The distribution of laurdan fluorescence GP values in domains of intensive fluorescence differed from the distribution of GP values for the whole membrane. This fact allows one to assume substantial differences in the microviscosity of intensively fluorescing domains and in the microviscosity of the rest membrane's part, and hence confirm the fact of existence of the nonhomogenieties. Meanwhile, in order to clarify this issue, further investigations with the application of other reliable techniques are needed.

Furthermore, it may be of interest to investigate the dependence of the organelle membranes on the complex of cell cytoskeleton elements. Such an investigation may be perspective from the viewpoint of redefining the functional role of all the cell's structural elements discussed above. 


\section{Acknowledgements}

The authors are grateful for the equipment granted by the Baikal Analytical Institution of Irkutsk Scientific Center (Siberian Branch, Russian Academy of Sciences).

\section{References}

[1] Sakuma, Y., Taniguchi, T., Kawakatsu, T. and Imai, M. (2013) Tubular Membrane Formation of Binary Giant Unilamellar Vesicles Composed of Cylinder and Inverse-Cone-Shaped Lipids. Biophysical Journal, 105, 2074-2081. https://doi.org/10.1016/j.bpj.2013.09.021

[2] Roux, A. (2013) The Physics of Membrane Tubes: Soft Templates for Studying Cellular Membranes. Soft Matter, 9, 6726-6736. https://doi.org/10.1039/c3sm50514f

[3] Ozolina, N.V., Nesterkina, I.S., Kolesnikova, E.V., Salyaev, R.K., Nurminsky, V.N., Rakevich, A. L., Martynovich, E.F. and Chernyshov, M.Yu. (2013) Tonoplast of Beta vulgaris L. Contains Detergent-Resistant Membrane Microdomains. Planta, 237, 859-871. https://doi.org/10.1007/s00425-012-1800-1

[4] Etxeberria, E., Gonzalez, P. and Pozueta-Romero, J. (2013) Architectural Remodeling of the Tonoplast during Fluid-Phase Endocytosis. Plant Signaling \& Behavior, 8, e24793. https://doi.org/10.4161/psb.24793

[5] Yoshida, K., Ohnishi, M., Fukao, Y., Okazaki, Y., Fujiwara, M., Song, C., Nakanishi, Y., Saito, K., Shimmen, T., Suzaki, T., Yayashi, F., Fukaki, H., Maeshima, M. and Mimura, T. (2013) Studies on Vacuolar Membrane Microdomains Isolated from Arabidopsis Suspension-Cultured Cells: Local Distribution of Vacuolar Membrane Proteins. Plant and Cell Physiology, 54, 1571-1584.

https://doi.org/10.1093/pcp/pct107

[6] Lingwood, D., Kaiser, H.J., Levental, I. and Simons, K. (2009) Lipid Rafts as Functional Heterogeneity in Cell Membranes. Biochemical Society Transactions, 37, 955-960. https://doi.org/10.1042/BST0370955

[7] Lingwood, D. and Simons, K. (2010) Lipid Rafts as a Membrane-Organizing Principle. Science, 327, 46-50. https://doi.org/10.1126/science.1174621

[8] Salyer, S.A., Olberding, J.R., Distler, A.A., Lederer, E.D., Clark, B.J., Delamere, N.A. and Khunfmiri, S.J. (2013) Vacuolar APTase Driven Potassium Transport in Highly Metastatic Breast Cancer Cells. Biochimica et Biophysica Acta, 1832, 1734-1743.

[9] Queirós, F., Fontes, N., Silva, P., Almeida, D., Maeshima, M., Gerós, H. and Fidalgo, F. (2009) Activity of Tonoplast Proton Pumps and $\mathrm{Na}^{+} / \mathrm{H}^{+}$Exchange in Potato Cell Cultures Is Modulated by Salt. Journal of Experimental Botany, 60, 1363-1374. https://doi.org/10.1093/jxb/erp011

[10] Janicka-Russak, M., Kabała, K., Młodzińska, E. and Kłobus, G. (2010) The Role of Polyamines in the Regulation of the Plasma Membrane and the Tonoplast Proton Pumps Under Salt Stress. The Journal of Plant Physiology, 167, 261-269. https://doi.org/10.1016/j.jplph.2009.09.010

[11] Wang, L., He, X., Zhao, Y., Shen, Y. and Huang, Z. (2011) Wheat Vacuolar $\mathrm{H}^{+}$-ATPase Subunit B Cloning and Its Involvement in Salt Tolerance. Planta, 234, 1-7. https://doi.org/10.1007/s00425-011-1383-2

[12] Zhang, M., Fang, Y., Liang, Z. and Huang, L. (2012) Enhanced Expression of Vacuolar $\mathrm{H}^{+}$-ATPase Subunit E in the Roots Is Associated with the Adaptation of Broussonetia papyrifera to Salt Stress. PLoS ONE, 7, e48183. https://doi.org/10.1371/journal.pone.0048183 
[13] Andreyev, I.M. (2001) Vacuole Functions in Higher Plant Cells. Fiziologiya Rastenii, 48, 777-787.

[14] Andreyev, I.M. (2012) The Role of Vacuole in Redox Homeostasis of Plant Cells. Fiziologiya Rastenii, 59, 660-667. https://doi.org/10.1134/s1021443712050032

[15] Sheahan, M.B., Rose, R.J. and McCurdy, D.W. (2007) Actin-Filament-Dependent Remodeling of the Vacuole in Cultured Mesophyll Protoplasts. Protoplasma, 230, 141-152. https://doi.org/10.1007/s00709-006-0236-5

[16] Assani, A., Moundanga, S., Beney, L. and Gervais, P. (2009) Vesicle Formation in the Membrane of Onion Cells (Allium cepa) during Rapid Osmotic Dehydration. Annals of Botany, 104, 1389-1395. https://doi.org/10.1093/aob/mcp256

[17] Nurmimsky, V.N., Ozolina, N.V., Nesterkina, I.S., Kolesnikova, E.V., Salyaev, R.K., Rakevich, A.L., Martynovich, E.F., Pilipchenko, A.A. and Chernyshov, M.Y. (2015) Peculiar Properties of Some Components in a Plant Cell Vacuole Morphological Structure Revealed by Confocal Microscopy. Cell and Tissue Biology, 9, 406-414. https://doi.org/10.1134/S1990519X15050090

[18] Loiseau, E., Schneider, J.A., Keber, F.C., Pelzl, C., Massiera, G., Salbreux, G. and Bausch, A.R. (2016) Shape Remodeling and Blebbing of Active Cytoskeletal Vesicles. Science Advances, 2, e1500465. https://doi.org/10.1126/sciadv.1500465

[19] Nurminsky, V.N., Chernyshov, M.Y., Ozolina, N.V., Nesterkina, I.S., Kolesnikova, E.V., Rakevich, A.L., Martynovich, E.F. and Salyaev, R.K. (2012) Detergent-Resistant Microdomains (Rafts) in Tonoplast. In: Rubin, A.B., Ed., Proceedings of 4 th Symposium of Russian Biophysicists Session 4. New Tendencies and Methods in Biophysics, University Publishing, Nizhnii Novgorod, 74.

[20] Gao, X.Q., Wang, X.L., Ren, F., Chen, J. and Wang, X.C. (2009) Dynamics of Vacuoles and Actin Filaments in Guard Cells and Their Roles in Stomatal Movement. Plant, Cell \& Environment, 32, 1108-1116. https://doi.org/10.1111/j.1365-3040.2009.01993.x

[21] Neuhaus, H.E. and Trentmann, O. (2014) Regulation of Transport Processes across the Tonoplast. Frontiers in Plant Science, 5, 460. https://doi.org/10.3389/fpls.2014.00460

[22] Salyaev, R.K., Kuzevanov, V.Y., Khaptagaev, V.Y. and Kopytchuk, V.N. (1981) Isolation and Purification of Vacuoles and Vacuolar Membranes from Plant Cells. Fiziologiya Rastenii, 28, 1295-1305.

[23] Gaus, K., Zech, T. and Harder, T. (2006) Visualizing Membrane Microdomains by Laurdan 2-Photon Microscopy. Molecular Membrane Biology, 23, 41-48. https://doi.org/10.1080/09687860500466857

[24] Baranov, S.I., Nurminsky, H.N. and Nurminsky, V.N. (2014) Automation of Fluorescent Microscopy Data Processing in Course of Measurement of Membrane Microviscosity. Proceedings of 27 th International Science Conference on Mathematical Methods in Engineering and Technology, Tambov, 175-178.

[25] Dabora, S.L. and Sheetz, M.P. (1988) The Microtubule-Dependent Formation of a Tubulovesicular Network with Characteristics of the ER from Cultured Cell Extracts. Cell, 54, 27-35. https://doi.org/10.1016/0092-8674(88)90176-6

[26] Reisen, D., Marty, F. and Leborgne-Castel, N. (2005) New Insights into the Tonoplast Architecture of Plant Vacuoles and Vacuolar Dynamics during Osmotic Stress. BMC Plant Biology, 5, 13. https://doi.org/10.1186/1471-2229-5-13

[27] Lollike, K. and Lindau, M. (1999) Membrane Capacitance Techniques to Monitor Granule Exocytosis in Neutrophils. Journal of Immunological Methods, 232, 111120. https://doi.org/10.1016/S0022-1759(99)00169-6 
[28] Farsad, K. and De Camilli, P. (2003) Mechanisms of Membrane Deformation. Current Opinion in Cell Biology, 15, 372-381. https://doi.org/10.1016/S0955-0674(03)00073-5

[29] Dautry-Vasart, A. and Luini, A. (2003) Membranes and Organelles. Current Opinion in Cell Biology, 15, 369-371. https://doi.org/10.1016/S0955-0674(03)00079-6

[30] Rustom, A., Saffrich, R., Markovic, I., Walther, P. and Gerdes, H.H. (2004) NanoTubular Highways for Intercellular Organelle Transport. Science, 303, 1007-1010. https://doi.org/10.1126/science.1093133

[31] Koster, G., VanDuijn, M., Hofs, B. and Dogterom, M. (2003) Membrane Tube Formation from Giant Vesicles by Dynamic Association of Motor Proteins. PNAS, 100, 15583-15588. https://doi.org/10.1073/pnas.2531786100

[32] Liu, A.P. and Fletcher, D.A. (2006) Actin Polymerization Serves as a Membrane Domain Switch in Model Lipid Bilayers. Biophysical Journal, 91, 4064-4070. https://doi.org/10.1529/biophysj.106.090852

[33] Neumann, A.K., Itano, M.S. and Jacobson, K. (2010) Understanding Lipid Rafts and Other Related Membrane Domains. F1000 Biology Reports, 2, 31. https://doi.org/10.3410/B2-31

[34] Oda, Y., Higaki, T., Hasezawa, S. and Kutsuna, N. (2009) New Insights into Plant Vacuolar Structure and Dynamics. International Review of Cell and Molecular Biology, 277, 103-135. https://doi.org/10.1016/S1937-6448(09)77003-0

[35] Duman, J.G., Pathak, N.J., Ladinsky, M.S., Mc Donald, K.L. and Forte, J.G. (2002) Three-Dimensional Reconstruction of Cytoplasmic Membrane Networks in Parietal Cells. Journal of Cell Science, 115, 1251-1258.

[36] Schneckenburger, H., Wagner, M., Kretzschmar, M., Strauss, W.S.L. and Sailer, R. (2004) Laser-Assisted Fluorescence Microscopy for Measuring Cell Membrane Dynamics. Photochemical \& Photobiological Sciences, 3, 817-822. https://doi.org/10.1039/b317047k

[37] Antonov, V.F., Chernysh, A.M., Pasechnik, V.I., Voznesensky, S.A. and Kozlova, E.K. (1999) Biophysics. Vlados Publising, Moscow, 288 p.

[38] Guillier, C., Cacas, J.-L., Recorbet, G., Depretre, N., Mounier, A., Mongrand, S., Simon-Plas, F., Wipf, D. and Dumas-Gaudot, E. (2014) Direct Purification of Detergent-Insoluble Membranes from Medicago truncatula Root Microsomes: Comparison between Flotation and Sedimentation. BMC Plant Biology, 14, 255.

https://doi.org/10.1186/s12870-014-0255-x

[39] Bagnat, M., Keranen, S., Shevchenko, A., Shevchenko, A. and Simons, K. (2000) Lipid Rafts Function in Biosynthetic Delivery of Proteins to the Cell Surface in Yeast. Proceedings of the National Academy of Sciences, 97, 3254-3259. https://doi.org/10.1073/pnas.97.7.3254

[40] Brown, D. (2002) Structure and Function of Membrane Rafts. International Journal of Medical Microbiology, 291, 433-437. https://doi.org/10.1078/1438-4221-00150

[41] Vladimirov, Y.A., Roshupkin, D.I., Potapenko, A.Y. and Deyev, A.I. (1983) Biophysics. Medicine Publishing, Moscow, 272 p.

[42] Boldyrev, A.A., Kyaivyaryainen, E.I. and Ilyuha, B.A. (2006) Biomembranology. Karelian Scientific Center of RAS Publishing, Petrozavodsk, 226 p.

[43] Los, D.A. and Murata, N. (2004) Membrane Fluidity and Its Role in the Perception of Environmental Signals. Biochimica et Biophysica Acta, 1666, 142-157. https://doi.org/10.1016/j.bbamem.2004.08.002 


\section{Acronyms}

ANS-8-anilino-1-naphthalene sulfonic acid

DPH-diphenilhexatrien

GP-generalized polarization

GUV_-giant unilamellar vesicle

FLIM-fluorescence lifetime imaging microscopy

LPM-lipid-protein microdomain

LPMN-lipid-protein membrane nanotube

MTS-membrane tubular structure

LPMN-lipid-protein membrane nanotube

SPAD-single-photon avalanche diode

V-H $\mathrm{H}^{+}$-ATPase-vacuolar $\mathrm{H}^{+}$-ATPase

Submit or recommend next manuscript to SCIRP and we will provide best service for you:

Accepting pre-submission inquiries through Email, Facebook, LinkedIn, Twitter, etc. A wide selection of journals (inclusive of 9 subjects, more than 200 journals)

Providing 24-hour high-quality service

User-friendly online submission system

Fair and swift peer-review system

Efficient typesetting and proofreading procedure

Display of the result of downloads and visits, as well as the number of cited articles Maximum dissemination of your research work

Submit your manuscript at: http://papersubmission.scirp.org/

Or contact abc@scirp.org 